\title{
Real Development Index
}

\section{Bartholomew $\mathbf{K}^{\star}$}

International Trade and Development, Xiamen, Fujian, China

\begin{abstract}
The Real Development Index (RDI) is a conglomerate index that measures and compares countries' level of development. The RDI takes into account various economic, social and environmental factors that other economic or human development indexes fail to do. Data for the index is relevant for the year 2014, and compares 110 countries using a variety of hard data and survey data. The index is comprised of three sections to measure a country's level of development: 1. Law and Stability, 2. Quality of Life, 3. Economic Development.
\end{abstract}

Keywords: Human development; Quality of life; Economic development; Development index

\section{Introduction}

In Beyond Economic Growth, published by the World Bank, the author states, "Human beings are at the center of concern for sustainable development. They are entitled to a healthy and productive life in harmony with nature." The author also states that development must include three aspects: economic, social and environmental. As of yet, there has not been a single index inclusive of all three to measure countries' level of development; this index hopes to achieve that in order to give an idea of countries' real level of development [1]. This author would also like to add that the idea of developed and underdeveloped is flawed, rather, all countries are developing because development is not an end goal. Development is the continuous improvement of a country in its standard and quality of living for its citizens. All countries face similar issues to varying degrees, thus a country's level of development can only be known relative to other countries' state of being.

\section{Index Configuration}

The RDI compared 110 countries with data relevant to the year 2014. The index consists of a total score of 100 points; a country can score up to 100 as the highest level of development compared to other countries. The RDI configures a country's score in three sections, with percentages added for the total score. The score card and factors are as followed:

Section one: Law and stability, $30 \%$

$$
\begin{array}{lll}
\text { 1. } & \text { Corruption } & 15 \% \\
\text { 2. } & \text { Peace and Security } & 7.5 \% \\
\text { 3. } & \text { Civil Liberties } & 7.5 \%
\end{array}
$$

Section two: Quality of life, $\mathbf{3 3 . 5 \%}$

$\begin{array}{lll}\text { 1. } & \text { Health } & 10 \% \\ \text { 2. } & \text { Environment } & 10 \% \\ \text { 3. } & \text { Education } & 7.5 \% \\ \text { 4. } & \text { Gender Equality } & 3 \% \\ \text { 5. } & \text { Community } & 3 \%\end{array}$

Section three: Economic development, 36.5\%

1. Standard of Living (unemployment $5.5 \%$, income inequality $5.5 \%$, average income $4 \%$, consumer quality $2.5 \%$ )

$\begin{array}{clc}\text { 2. } & \text { Financial Market Development } & 5 \% \\ \text { 3. } & \text { Infrastructure } & 5 \% \\ \text { 4. } & \text { Business Sophistication } & 3 \% \\ \text { 5. } & \text { Technology } & 3 \% \\ \text { 6. } & \text { Innovation } & 3 \%\end{array}$

\section{Factors}

\section{Corruption}

Is taken from Transparency International's 2013 Corruptions Perception Index (CPI). The CPI ranks countries "by their perceived levels of corruption, as determined by expert assessments and opinion surveys." The definition of corruption in this index is "the misuse of public power for private gain [2]" (Table 1).

\section{Peace}

Is taken from the Institute for Economics and Peace's 2014 Global Peace Index. The GPI ranks countries according to their peacefulness. It takes into account 22 factors: 1 . Number of external and internal conflicts fought, 2. Number of deaths from organized conflict (external), 3. Number of deaths from organized conflict (internal), 4. Level of organized conflict (internal), 5. Relations with neighboring countries, 6. Level of perceived criminality in society, 7. Number of refugees and displaced persons as percentage of population, 8 . Political instability, 9. Terrorist activity, 10. Political terror scale, 11. Number of homicides per 100,000 people, 12. Level of violent crime, 13. Likelihood of violent demonstrations, 14 . Number of jailed persons per 100,000 people, 15 . Number of internal security officers and police per 100,000 people, 16. Military expenditure as a percentage of GDP, 17. Number of armed-services personal, 18. Volume of transfers of

${ }^{*}$ Corresponding author: Bartholomew K, International Trade and Development, Xiamen, Fujian, China, E-mail: KyleBartholomew@my.unt.edu

Received October 08, 2014; Accepted March 03, 2015; Published March 13, 2015

Citation: Bartholomew K (2015) Real Development Index. J Glob Econ 3: 135 doi:10.4172/2375-4389.1000135

Copyright: ( 92015 Bartholomew K. This is an open-access article distributed under the terms of the Creative Commons Attribution License, which permits unrestricted use, distribution, and reproduction in any medium, provided the original author and source are credited. 


\begin{tabular}{|l|c|}
\hline Country & Score: $\mathbf{8 7 . 0 2 4 5}$ \\
\hline Switzerland & \\
\hline Ranking1 of 110 & $\mathbf{2 7 . 4 8 \%}$ \\
\hline Section One & 12.75 \\
\hline Corruption & 7.23 \\
\hline Peace and Stability & $\mathbf{7 . 5}$ \\
\hline Civil Liberties & $\mathbf{2 9 . 6 9 \%}$ \\
\hline Section Two & 9.46 \\
\hline Health & 8.767 \\
\hline Environment & 6.45 \\
\hline Education & 2.31 \\
\hline Gender Equality & 2.703 \\
\hline Community & $\mathbf{2 9 . 8 5 4 5 \%}$ \\
\hline Section Three & 14.8645 \\
\hline Standard of Living & 3.6 \\
\hline Financial Market Development & 4.4 \\
\hline Infrastructure & 2.25 \\
\hline Business Sophistication & 2.37 \\
\hline Technology & 2.37 \\
\hline Innovation & \\
\hline
\end{tabular}

Table 1: Comparision of Sections.

major conventional weapons as recipient (imports) per 100,000 people, 19. Volume of transfers of major conventional weapons as supplier (exports) per 1000,000 people, 20. Financial contributions to UN peace keeping missions, 21. Nuclear and heavy weapons capability, 22. Ease of access to small arms and light weapons [3].

\section{Civil liberties}

Is taken from the civil liberties section of the 2014 Freedom of the World survey [4].

\section{Health}

Is life expectancy at time of birth. Data is taken from the World Bank [5].

\section{Environment}

Is the 2014 Environmental Performance Index, which "ranks countries on performance indicators tracked across policy categories that cover both environmental public health and ecosystem vitality" [6].

\section{Education}

Is taken from the 2013-14 Global Competitiveness Report and includes six factors: 1 . quality of primary education, 2 . quality of the educational system, 3. quality of math and science education, 4 . quality of management schools, 5. availability of research and training services, 6. extent of staff training [7].

\section{Gender equality}

Is taken from the 2013 Global Gender Gap Index. "It ranks countries according to their gender equality rather than woman's empowerment." It measures four categories: Economic participation and opportunity, educational attainment, health and survival, and political empowerment [8].

\section{Community}

Is taken from Charities Aid Foundation's 2013 World Giving Index. The GPI is based on Gallup Poll questions, which had 500-2000 participants per country. The questions regard donating to charity, volunteering time to an organization and helping a stranger [9].

\section{Unemployment}

Is unemployment total $\%$ of the labor force; the share of the labor force that is without work but available for and seeking unemployment. Data is taken from the World Bank [10].

\section{Income inequality}

Is Distribution of Family Income- GINI Index. Data is taken from various sources. There is a lack of quantifiable data for Chad, Oman, Saudi Arabia, Lebanon and United Arab Emirates [11-19].

\section{Average income}

Is GDP PPP Per Capita. GDP per capita is gross domestic product divided by mid-year population. GDP PPP Per Capita is GDP per capita at purchasing power parity. Data is taken from the World Bank [20,21].

\section{Consumer quality}

Is taken from the 2013-14 Global Competitiveness Report and includes three factors: 1 . degree of customer orientation, 2 . buyer sophistication, 3. extent of marketing. This factor was included to show a citizen's access to, and awareness of products in the market, which enables them to live a healthier material life [7].

\section{Financial market development}

Is taken from the 2013-14 Global Competitiveness Report and includes seven factors: 1 . availability of financial services, 2 . affordability of financial services, 3 . financing through local equity market, 4 . ease of access to loans, 5 . venture capital availability, 6 . soundness of banks, 7 . regulation of securities exchange [7].

\section{Infrastructure}

Is taken from the 2013-14 Global Competitiveness Report and includes five factors: 1. quality of roads, 2 . quality of railroad infrastructure, 3. quality of port infrastructure, 4. quality of transport infrastructure, 5. quality of electricity supply [7].

\section{Business sophistication}

Is taken from the 2013-14 Global Competitiveness Report and includes twelve factors: 1 . local supplier quality, 2. local supplier quantity, 3. state of cluster development, 4. value chain breadth, 5 .

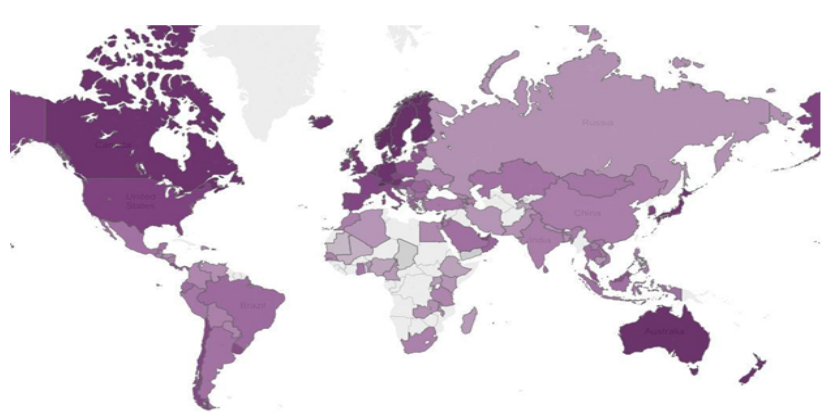

Figure 1: Ranking and scores(Note: The darker the shade of purple the more developed the country is, the lighter the shade of purple the less developed the country is, excluding those where no data was collected for. Map created in Tableau Public). 
1. Switzerland, 87.0245

2. Norway, 85.684

3. Sweden, 82.9955

4. Finland, 82.814

13. Denmark, 80.257

14. Singapore, 78.9485

15. Japan, 78.3835

16. Belgium, 76.3395

17. United Kingdom, 75.4395

18. Ireland, 74.4785

19. United States, 70.3735

20. Qatar, 69.3515

21. South Korea, 69.350

22. Spain, 69.294

23. France, 68.851

24. Slovenia, 68.8395

25. Czech Republic, 68.407

26. Cyprus, 67.8245

27. Chile, 67.8245

28. Estonia, 66.8395

29.Portugal, 66.7175

30. Italy, 66.696

31. United Arab Emirates, 66.479

32. Uruguay, 65.1405

33. Israel, 64.394

34. Poland, 63.898

35. Malaysia, 62.7715

36. Hungary, 62.346

37. Mauritius, 62.021

38. Costa Rica, 61.722

39. Lithuania, 61.317

40. Panama, 59.5655

41. Croatia, 58.39

42. Latvia, 58.3865

43. Greece, 55.914

44. Oman, 55.4485

45. Trinidad \& Tobago, 55.353

46. Romania, 54.385
5. Luxembourg, 82.1915

6. Australia, 81.59

7. New Zealand, 81.2695

8. Iceland, 81.172

47. Brazil, 53.383

48. Thailand, 53.271

49. Ecuador, 53.0665

50. Sri Lanka, 52.784

51. Saudi Arabia, 51.7385

52. Indonesia, 51.367

53. Bulgaria, 51.2995

54. Argentina, 51.061

55. Mexico, 50.985

56. Kazakhstan, 50.2165

57. Ghana, 49.279

58. Mongolia, 49.073

59. Vietnam, 48.993

60. Peru, 48.988

61. Jordan, 48.8885

62. Macedonia, 48.597

63. Serbia, 48.5445

64. Turkey, 48.368

65. Philippines, 47.6285

66. Armenia, 47.4635

67. Nicaragua, 47.143

68. Guatemala, 47.117

69. Laos, 47.107

70. Moldova, 46.9495

71. India, 46.782

72. El Salvador, 46.5995

73. China, 46.390

74. Morocco, 46.2555

75. Bolivia, 46.0785

76. South Africa, 45.5605

77. Albania, 45.526

78. Azerbaijan, 45.5025

79. Ukraine, 45.4105

80. Tanzania, 44.8035
9. Germany, 80.9245

10. Netherlands, 80.873

11. Canada, 80.5605

12. Austria, 80.2955

81. Paraguay, 44.4725

82. Senegal, 44.380

83. Dominican Republic, 44.2405

84. Colombia, 44.020

85. Georgia, 43.5315

86. Lebanon, 43.3745

87. Egypt, 42.5345

88. Nepal, 42.089

89. Honduras, 42.0415

90. Venezuela, 41.6035

91. Malawi, 41.3415

92. Russia, 41.2030

93. Bangladesh, 41.2105

94. Zambia, 41.0055

95. Pakistan, 40.790

96. Cambodia, 40.5805

97. Iran, 39.822

98. Algeria, 39.684

99. Benin, 39.4735

100. Kenya, 39.3605

101. Cameroon, 38.352

102. Madagascar, 38.337

103. Uganda, 37.9985

104. Tajikistan, 36.396

105. Ethiopia, 36.241

106. Nigeria, 35.595

107. Mali, 32.278

108. Mauritania, 30.298

109. Yemen, 26.1205

110. Chad, 24.9755

Table 2: Country Score Card Example.

\begin{tabular}{|c|c|c|c|c|c|c|}
\hline Americas & $\begin{array}{l}\text { Northern and Western } \\
\text { Europe }\end{array}$ & $\begin{array}{l}\text { Southern and } \\
\text { Eastern Europe }\end{array}$ & Oceania and East Asia & $\begin{array}{l}\text { Central and South } \\
\text { Asia }\end{array}$ & $\begin{array}{l}\text { Middle East and } \\
\text { North Africa }\end{array}$ & Sub-Saharan Africa \\
\hline 1. Iceland & 1. Switzerland & 1. Spain & 1. Australia & 1. Sri Lanka & 1. Qatar & 1. Mauritius \\
\hline 2. Canada & 2. Norway & 2. Slovenia & 2. New Zealand & 2. Kazakhstan & 2. United Arab Emirates & 2. Ghana \\
\hline 3. United States & 3. Sweden & 3. Czech Republic & 3. Singapore & 3. Armenia & 3. Israel & 3. South Africa \\
\hline 4. Chile & 4. Finland & 4. Cyprus & 4. Japan & 4. India & 4. Oman & 4. Tanzania \\
\hline 5. Uruguay & 5. Luxembourg & 5. Estonia & 5. South Korea & 5. Azerbaijan & 5. Saudi Arabia & 5. Senegal \\
\hline 6. Costa Rica & 6. Germany & 6. Portugal & 6. Malaysia & 6. Georgia & 6. Jordan & 6. Malawi \\
\hline 7. Panama & 7. Netherlands & 7. Italy & 7. Thailand & 7. Nepal & 7. Morocco & 7. Zambia \\
\hline 8. Trinidad \& Tobago & 8. Austria & 8. Poland & 8. Indonesia & 8. Bangladesh & 8. Lebanon & 8. Benin \\
\hline 9. Brazil & 9. Denmark & 9. Hungary & 9. Mongolia & 9. Pakistan & 9. Egypt & 9. Kenya \\
\hline 10. Ecuador & 10. Belgium & 10. Lithuania & 10. Vietnam & 10. Tajikistan & 10. Iran & 10. Cameroon \\
\hline 11. Argentina & 11. United Kingdom & 11. Croatia & 11.phillippines & & 11. Algeria & 11. Madagascar \\
\hline 12. Mexico & 12. Ireland & 12. Latvia & 12. Laos & & 12. Yemen & 12. Uganda \\
\hline 13. Peru & 13. France & 13. Greece & 13. China & & & 13. Ethiopia \\
\hline 14. Nicaragua & & 14. Romania & 14. Cambodia & & & 14. Nigeria \\
\hline 15. Guatemala & & 15. Bulgaria & & & & 15. Mali \\
\hline 16. Elsalvador & & 16. acedonia & & & & 16. Mauritania \\
\hline 17. Bolivia & & 17. Serbia & & & & 17. Chad \\
\hline 18. Paraguay & & 18. Turkey & & & & \\
\hline
\end{tabular}




\begin{tabular}{|c|c|c|c|c|c|c|}
\hline $\begin{array}{l}\text { 19. Dominican } \\
\text { Republic }\end{array}$ & & 19. Moldova & & & & \\
\hline 20. Colombia & & 20. Albania & & & & \\
\hline 21. Honduras & & 21. Ukraine & & & & \\
\hline 22. Venezuela & & 22. Russia & & & & \\
\hline Avg: 54.65959 & Avg: 79.8590 & Avg: 57.5521 & Avg: 59.7306 & Avg: 44.6789 & Avg: 49.5075 & Avg: 40.0776 \\
\hline
\end{tabular}

Table 3: Ranking and Scores.

Countries by Region, Rank Order and Region Average.

\begin{tabular}{|c|c|c|}
\hline $\begin{array}{l}2014 \text { Real Development } \\
\text { Index }\end{array}$ & $\begin{array}{l}2014 \text { Human Development } \\
\text { Index21 }\end{array}$ & GNI per capita PPP22 \\
\hline 1. Switzerland & 1. Norway & 1. Qatar \\
\hline 2. Norway & 2. Australia & 2. Singapore \\
\hline 3. Sweden & 3. Switzerland & 3. Norway \\
\hline 4. Finland & 4. Netherlands & 4. United Arab Emirates \\
\hline 5. Luxembourg & 5. United States of America & 5. Switzerland \\
\hline 6. Australia & 6. Germany & 6. Luxembourg \\
\hline 7. New Zealand & 7. New Zealand & 7. United States \\
\hline 8. Iceland & 8. Canada & 8. Saudi Arabia \\
\hline 9. Germany & 9. Singapore & 9. Oman \\
\hline 10. Netherlands & 10. Denmark & 10. Sweden \\
\hline 11. Canada & 11. Ireland & 11. Netherlands \\
\hline 12. Austria & 12. Sweden & 12. Germany \\
\hline 13. Denmark & 13. Iceland & 13. Austria \\
\hline 14. Singapore & 14. United Kingdom & 14. Denmark \\
\hline 15. Japan & 15. South Korea & 15. Canada \\
\hline 16. Belgium & 16. Japan & 16. Australia \\
\hline 17. United Kingdom & 17. Israel & 17. Belgium \\
\hline 18. Ireland & 18. France & 18. Iceland \\
\hline 19. United States & 19. Austria & 19. Finland \\
\hline 20. Qatar & 20. Belgium & 20. Ireland \\
\hline 21. South Korea & 21. Luxembourg & 21. France \\
\hline 22. Spain & 22. Finland & 22. United Kingdom \\
\hline 23. France & 23. Slovenia & 23. Japan \\
\hline 24. Slovenia & 24. Italy & 24. Italy \\
\hline 25. Czech Republic & 25. Spain & 25. South Korea \\
\hline 26. Cyprus & 26. Czech Republic & 26. Spain \\
\hline 27. Chile & 27. Greece & 27. New Zealand \\
\hline 28. Estonia & 28. Qatar & 28. Israel \\
\hline 29. Portugal & 29. Cyprus & 29.Slovenia \\
\hline 30. Italy & 30. Estonia & 30. Cyprus \\
\hline 31. United Arab Emirates & 31. Saudi Arabia & 31. Czech Republic \\
\hline 32. Uruguay & 32. Lithuania & 32. Trinidad \& Tobago \\
\hline 33. Israel & 33. Poland & 33. Portugal \\
\hline 34. Poland & 34. United Arab Emirates & 34. Greece \\
\hline 35. Malaysia & 35. Chile & 35. Estonia \\
\hline 36. Hungary & 36. Portugal & 36. Lithuania \\
\hline 37. Mauritius & 37. Hungary & 37. Russia \\
\hline 38. Costa Rica & 38. Croatia & 38. Hungary \\
\hline 39. Lithuania & 39. Latvia & 39. Poland \\
\hline 40. Panama & 40. Argentina & 40. Malaysia \\
\hline 41. Croatia & 41. Urugay & 41. Latvia \\
\hline 42. Latvia & 42. Romanuia & 42. Chile \\
\hline 43. Greece & 43.Oman & 43. Croatia \\
\hline 44. Oman & 44. Russia & 44. Kazakhstan \\
\hline 45. Trinidad \& Tobago & 45. Bulgaria & 45. Panama \\
\hline 46. Romania & 46. Malaysia & 46. Uruguay \\
\hline 47. Brazil & 47. Mauritius & 47. Turkey \\
\hline 48. Thailand & 48. Trinidad \& Tobago & 48. Romania \\
\hline 49. Ecuador & 49. Lebanon & 49. Venezuela \\
\hline 50. Sri Lanka & 50. Panama & 50. Lebanon \\
\hline 51. Saudi Arabia & 51. Venezuela & 51. Mauritius \\
\hline
\end{tabular}

\begin{tabular}{|c|c|c|}
\hline 52. Indonesia & 52. Costa Rica & 52. Azerbaijan \\
\hline 53. Bulgaria & 53. Turkey & 53. Mexico \\
\hline 54. Argentina & 54. Kazakhstan & 54. Iran \\
\hline 55. Mexico & 55. Mexico & 55. Bulgaria \\
\hline 56. Kazakhstan & 56. Sri Lanka & 56. Brazil \\
\hline 57. Ghana & 57. Iran & 57. Costa Rica \\
\hline 58. Mongolia & 58. Azerbaijan & 58. Thailand \\
\hline 59. Vietnam & 59. Jordan & 59. Algeria \\
\hline 60. Peru & 60. Serbia & 60. Serbia \\
\hline 61. Jordan & 61. Brazil & 61. South Africa \\
\hline 62. Macedonia & 62. Georgia & 62. Colombia \\
\hline 63. Serbia & 63. Peru & 63. China \\
\hline 64. Turkey & 64. Ukraine & 64. Jordan \\
\hline 65. Philippines & 65. Macedonia & 65. Dominican Republic \\
\hline 66. Armenia & 66. Armenia & 66. Macedonia \\
\hline 67. Nicaragua & 67. Thailand & 67. Peru \\
\hline 68. Guatemala & 68 China & 68. Egypt \\
\hline 69. Laos & 69. Algeria & 69. Ecuador \\
\hline 70. Moldova & 70. Albania & 70. Albania \\
\hline 71. India & 71. Colombia & 71. Sri Lanka \\
\hline 72. EI Salvador & 72. Ecuador & 72. Indonesia \\
\hline 73. China & 73. Dominican Republic & 73. Ukraine \\
\hline 74. Morocco & 74. Mongolia & 74. Mongolia \\
\hline 75. Bolivia & 75. Indonesia & 75. Armenia \\
\hline 76. South Africa & 76. Egypt & 76. Philippines \\
\hline 77. Albania & 77. Paraguay & 77. Paraguay \\
\hline 78. Azerbaijan & 78. Bolivia & 78. El Salvador \\
\hline 79. Ukraine & 79. Moldova & 79. Guatemala \\
\hline 80. Tanzania & 80. El Salvador & 80. Georgia \\
\hline 81. Paraguay & 81. Philippines & 81. Morocco \\
\hline 82. Senegal & 82. South Africa & 82. Bolivia \\
\hline 83. Dominican Republic & 83.Vietnam & 83. Nigeria \\
\hline 84. Colombia & 84. Guatemala & 84. India \\
\hline 85. Georgia & 85. Honduras & 85. Moldova \\
\hline 86. Lebanon & 86. Morocco & 86. Vietnam \\
\hline 87. Egypt & 87. Nicaragua & 87. Pakistan \\
\hline 88. Nepal & 88. Tajikistan & 88. Laos \\
\hline 89. Honduras & 89. India & 89. Nicaragua \\
\hline 90. Venezuela & 90. Cambodia & 90. Honduras \\
\hline 91. Malawi & 91. Ghana & 91. Ghana \\
\hline 92. Russia & 92.Laos & 92. Yemen \\
\hline 93. Bangladesh & 93. Zambia & 93. Zambia \\
\hline 94 Zambia & 94. Bangladesh & 94. Bangladesh \\
\hline 95. Pakistan & 95. Nepal & 95. Cambodia \\
\hline 96. Cambodia & 96. Pakistan & 96. Mauritania \\
\hline 97. Iran & 97. Kenya & 97. Kenya \\
\hline 98. Algeria & 98. Cameroon & 98. Cameroon \\
\hline 99. Benin & 99. Nigeria & 99. Tajikistan \\
\hline 100. Kenya & 100.Yemen & 100. Nepal \\
\hline 101. Cameroon & 101. Madagascar & 101. Senegal \\
\hline 102. Madagascar & 102. Tanzania & 102. Chad \\
\hline 103. Uganda & 103. Mauritania & 103. Benin \\
\hline 104. Tajikistan & 104. Senegal & 104. Tanzania \\
\hline
\end{tabular}




\begin{tabular}{|c|c|c|}
\hline 105. Ethiopia & 105. Uganda & 105. Mali \\
\hline 106. Nigeria & 106. Benin & 106. Uganda \\
\hline 107. Mali & 107.Ethiopia & 107. Ethiopia \\
\hline 108. Mauritania & 108. Malawi & 108. Madagascar \\
\hline 109. Yemen & 109. Mali & 109. Malawi \\
\hline 110. Chad & 110. Chad & NA Argentina \\
\hline $\begin{array}{l}2014 \text { Real Development } \\
\text { Index }\end{array}$ & $\begin{array}{l}2014 \text { Human Development } \\
\text { Index21 }\end{array}$ & GNI per capita PPP22 \\
\hline 1. Switzerland & 1. Norway & 1. Qatar \\
\hline 2. Norway & 2. Australia & 2. Singapore \\
\hline 3. Sweden & 3. Switzerland & 3. Norway \\
\hline 4. Finland & 4. Netherlands & 4. United Arab Emirates \\
\hline 5. Luxembourg & 5. United States of America & 5. Switzerland \\
\hline 6. Australia & 6. Germany & 6. Luxembourg \\
\hline 7. New Zealand & 7. New Zealand & 7. United States \\
\hline 8. Iceland & 8. Canada & 8. Saudi Arabia \\
\hline 9. Germany & 9. Singapore & 9. Oman \\
\hline 10. Netherlands & 10. Denmark & 10. Sweden \\
\hline 11. Canada & 11.Ireland & 11. Netherlands \\
\hline 12. Austria & 12. Sweden & 12. Germany \\
\hline 13. Denmark & 13. Iceland & 13. Austria \\
\hline 14. Singapore & 14. United Kingdom & 14. Denmark \\
\hline 15. Japan & 15. South Korea & 15. Canada \\
\hline 16. Belgium & 16. Japan & 16. Australia \\
\hline 17. United Kingdom & & 17. Belgium \\
\hline 18. Ireland & 18. France & 18. Iceland \\
\hline 19. United States & 19. Austria & 19. Finland \\
\hline 20. Qatar & 20. Belgium & 20. Ireland \\
\hline 21. South Korea & 21. Luxembourg & 21. France \\
\hline 22. Spain & 22. Finland & 22. United Kingdom \\
\hline 23. France & 23. Slovenia & 23. Japan \\
\hline 24. Slovenia & 24. Italy & 24. Italy \\
\hline 25. Czech Republic & 25. Spain & 25. South Korea \\
\hline 26. Cyprus & 26. Czech Republic & 26. Spain \\
\hline 27. Chile & 27. Greece & 27. New Zealand \\
\hline 28. Estonia & 28. Qatar & 28. Israel \\
\hline 29. Portugal & 29. Cyprus & 29.Slovenia \\
\hline 30. Italy & 30. Estonia & 30. Cyprus \\
\hline 31. United Arab Emirates & 31. Saudi Arabia & 31. Czech Republic \\
\hline 32. Uruguay & 32. Lithuania & 32. Trinidad \& Tobago \\
\hline 33. Israel & 33. Poland & 33. Portugal \\
\hline 34. Poland & 34. United Arab Emirates & 34. Greece \\
\hline 35. Malaysia & 35. Chile & 35. Estonia \\
\hline 36. Hungary & 36. Portugal & 36. Lithuania \\
\hline 37. Mauritius & 37. Hungary & 37. Russia \\
\hline 38. Costa Rica & 38. Croatia & 38. Hungary \\
\hline 39. Lithuania & 39. Latvia & 39. Poland \\
\hline 40. Panama & 40. Argentina & 40. Malaysia \\
\hline 41. Croatia & 41. Uruguay & 41. Latvia \\
\hline 42. Latvia & 42. Romania & 42. Chile \\
\hline 43. Greece & 43. Oman & 43. Croatia \\
\hline 44. Oman & 44. Russia & 44. Kazakhstan \\
\hline 45. Trinidad \& Tobago & 45. Bulgaria & 45. Panama \\
\hline 46. Romania & 46. Malaysia & 46. Uruguay \\
\hline 47. Brazil & 47. Mauritius & 47. Turkey \\
\hline 48. Thailand & 48.Trinidad \& Tobago & 48. Romania \\
\hline 49. Ecuador & 49. Lebanon & 49. Venezuela \\
\hline 50. Sri Lanka & 50. Panama & 50. Lebanon \\
\hline 51. Saudi Arabia & 51. Venezuela & 51. Mauritius \\
\hline 52. Indonesia & 52. Costa Rica & 52. Azerbaijan \\
\hline 53. Bulgaria & 53. Turkey & 53. Mexico \\
\hline 54. Argentina & 54. Kazakhstan & 54. Iran \\
\hline
\end{tabular}

\begin{tabular}{|c|c|c|}
\hline 55. Mexico & 55. Mexico & 55. Bulgaria \\
\hline 56. Kazakhstan & 56. Sri Lanka & 56. Brazil \\
\hline 57. Ghana & 57. Iran & 57. Costa Rica \\
\hline 58. Mongolia & 58. Azerbaijan & 58. Thailand \\
\hline 59. Vietnam & 59. Jordan & 59. Algeria \\
\hline 60. Peru & 60. Serbia & 60. Serbia \\
\hline 61. Jordan & 61. Brazil & 61. South Africa \\
\hline 62. Macedonia & 62. Georgia & 62. Colombia \\
\hline 63. Serbia & 63. Peru & 63. China \\
\hline 64. Turkey & 64. Ukraine & 64. Jordan \\
\hline 65. Philippines & 65. Macedonia & 65. Dominican Republic \\
\hline 66. Armenia & 66.Armenia & 66. Macedonia \\
\hline 67. Nicaragua & 67. Thailand & 67. Peru \\
\hline 68. Guatemala & 68. China & 68. Egypt \\
\hline 69. Laos & 69. Algeria & 69. Ecuador \\
\hline 70. Moldova & 70. Albania & 70. Albania \\
\hline 71. India & 71. Colombia & 71. Sri Lanka \\
\hline 72. El Salvador & 72. Ecuador & 72. Indonesia \\
\hline 73. China & 73. Dominican Republic & 73. Ukraine \\
\hline 74. Morocco & 74. Mongolia & 74. Mongolia \\
\hline 75. Bolivia & 75. Indonesia & 75. Armenia \\
\hline 76. South Africa & 76. Egypt & 76. Philippines \\
\hline 77. Albania & 77. Paraguay & 77. Paraguay \\
\hline 78. Azerbaijan & 78. Bolivia & 78. El Salvador \\
\hline 79. Ukraine & 79. Moldova & 79. Guatemala \\
\hline 80. Tanzania & 80. El Salvador & 80. Georgia \\
\hline 81. Paraguay & 81. Philippines & 81. Morocco \\
\hline 82. Senegal & 82. South Africa & 82. Bolivia \\
\hline 83. Dominican Republic & 83. Vietnam & 83. Nigeria \\
\hline 84. Colombia & 84. Guatemala & 84. India \\
\hline 85. Georgia & 85. Honduras & 85. Moldova \\
\hline 86. Lebanon & 86. Morocco & 86. Vietnam \\
\hline 87. Egypt & 87. Nicaragua & 87. Pakistan \\
\hline 88. Nepal & 88. Tajikistan & 88. Laos \\
\hline 89. Honduras & 89. India & 89. Nicaragua \\
\hline 90. Venezuela & 90. Cambodia & 90. Honduras \\
\hline 91. Malawi & 91. Ghana & 91. Ghana \\
\hline 92. Russia & 92.Laos & 92. Yemen \\
\hline 93. Bangladesh & 93. Zambia & 93. Zambia \\
\hline 94. Zambia & 94.Bangladesh & 94. Bangladesh \\
\hline 95. Pakistan & 95. Nepal & 95. Cambodia \\
\hline 96. Cambodia & 96. Pakistan & 96. Mauritania \\
\hline 97. Iran & 97. Kenya & 97. Kenya \\
\hline 98. Algeria & 98. Cameroon & 98. Cameroon \\
\hline 99. Benin & 99. Nigeria & 99. Tajikistan \\
\hline 100. Kenya & 100. Yemen & 100. Nepal \\
\hline 101. Cameroon & 101. Madagascar & 101. Senegal \\
\hline 102. Madagascar & 102. Tanzania & 102. Chad \\
\hline 103. Uganda & 103. Mauritania & 103. Benin \\
\hline 104. Tajikistan & 104. Senegal & 104. Tanzania \\
\hline 105. Ethiopia & 105. Uganda & 105. Mali \\
\hline 106. Nigeria & 106. Benin & 106. Uganda \\
\hline 107. Mali & 107. Ethiopia & 107. Ethiopia \\
\hline 108. Mauritania & 108. Malawi & 108. Madagascar \\
\hline 109. Yemen & 109. Mali & 109. Malawi \\
\hline 110. Chad & 110. Chad & NA Argentina \\
\hline
\end{tabular}

Table 4: Real Development Index, Human Development Index, GNI per capita PPP Compared. 
production process sophistication, 6 . willingness to delegate authority, 7. effectiveness of anti-monopoly policy, 8. ethical behavior of firms, 9 . strength of auditing, 10. Efficiency of corporate boards, 11. protection of minority shareholders' interest, 12. Strength of investor protection [7].

\section{Technology}

Is taken from the 2013-14 Global competitiveness report and includes four factors: 1 . availability of latest technologies, 2. firm level technology absorption, 3. FDI and technology transfer, 4. availability of scientists and engineers [7] (Figure 1 and Tables 2-4).

\section{Innovation}

It is taken from the 2013-14 Global competitiveness report and includes five factors:

1. Capacity for innovation,

2. Quality of scientific research institutions,

3. Company spending on $R \& D$,

4. University-industry collaboration,

5. Government procurement of advanced tech products [7].

\section{References}

1. Soubbotina, Tatyana (2004) "Beyond Economic Growth." Business and Economics: World Bank. 2014.

2. Corruption Perceptions Index (2014) Transparency International.

3. Global Peace Index (2014) Institute for Economics and Peace.

4. "Freedom in the World 2015" (2015) World Freedom Report.
5. Life Expectancy at Birth (2013) Life Expectancy at Birth. World Bank.

6. Environmental Performance Index (2014) Yale University, New Haven, United States.

7. Martin, Xavier SI, Elsa VA (2014) Global Competitiveness Report. Global Competitiveness Report. World Economic Forum.

8. Global Gender Gap (2013) Global Gender Gap. World Economic Forum.

9. World Giving Index (2013) Charities Aid Foundation

10. Unemployment (2013) Total (\% of Total Labor Force) Unemployment. World Bank.

11. "Income GINI Coefficient" (2013) Human Development Reports. World Bank.

12. Household Income or Consumption by Percentage of Share (2013) The World Factbook. Central Intelligence Agency.

13. GINI Index (2014) GINI Index. World Bank.

14. "The Poor Getting Poorer in Oman" (2013) Al Bawaba. Muscat Press and Publishing House.

15. "Oman-Poverty and Wealth" (2014) Nations Encyclopedia. Nations Encyclopedia.

16. Poverty, Growth and Income Distribution in Lebanon (2008) United Nations Development Programme United Nations Development Programme.

17. "Saudi Arabia Poverty and Wealth, Information about Poverty and Wealth in Saudi Arabia" (2014) Nations Encyclopedia.

18. "United Arab Emirates Poverty and Wealth"(2014) Encyclopedia of the Nations.

19. "GDP per Capita (2014) PPP (current International \$)." GDP per Capita PPP. World Bank.

20. "Human Development Report" (2014) Human Development Report. United Nations Development Programme.

21. "GNI per Capita, PPP”(2015) World Bank Data. World Bank. 